\title{
Rotational excitation of SiS molecules by collisions with He atoms
}

\author{
L. F. M. Vincent ${ }^{1,2}$, A. Spielfiedel ${ }^{1}$, and F. Lique ${ }^{1,3}$ \\ 1 LERMA and UMR 8112 of CNRS, Observatoire de Paris-Meudon, 92195 Meudon Cedex, France \\ e-mail: francois.lique@obspm.fr \\ 2 DAMIR, Instituto de Estructura de la Materia, CSIC, Calle Serrano 121, 28006 Madrid, Spain \\ 3 Department of Chemistry and Biochemistry, University of Maryland, College park, Maryland 20742-2021, USA
}

Received 14 May 2007 / Accepted 21 June 2007

\begin{abstract}
Context. Observations of molecular emission at millimeter and infrared wavelengths, supplemented by careful and detailed modeling, are powerful tools for investigating the physical and chemical conditions of astrophysical objects. Modeling of molecular emission requires excitation calculations using radiative as well as collisional rates with the most abundant species.

Aims. The present paper focuses on the calculation of rate coefficients among the first rotational levels of the SiS molecule in its ground vibrational state in collision with $\mathrm{He}$.

Methods. A new accurate two dimensional potential energy surface system was calculated for the SiS-He system using highly correlated ab initio calculations. Dynamical calculations of pure rotational (de)excitation of SiS by collisions with He were performed for the first rotational levels using the near exact close-coupling approach.

Results. Collisional cross sections among the 26 first rotational levels of SiS were calculated for kinetic energies up to $1500 \mathrm{~cm}^{-1}$. Rate coefficients between the rotational levels were calculated for temperatures ranging from 5 to $200 \mathrm{~K}$. A propensity rule that favors even $\Delta j$ transitions is found and is explained by the small asymmetry of the SiS-He potential energy surface.
\end{abstract}

Key words. ISM: molecules - molecular data - molecular processes

\section{Introduction}

The first detection of SiS was reported more than 30 years ago by Morris et al. (1975). SiS was detected through the $j=6 \rightarrow 5$ and $j=5 \rightarrow 4$ rotational line emission toward the IRC +10216 circumstellar envelope. SiS has been extensively observed, in particular toward the C-rich star IRC +10216 (Olofsson et al. 1982; Henkel et al. 1983; Bieging \& Nguyen-Quang-Rieu 1989; Fonfría Expósito et al. 2006). Maser emission was first pointed out by Henkel et al. (1983) in the $j=1 \rightarrow 0$ rotational transition in the $v=0,1$ and 2 vibrational states and recently by Fonfría Expósito et al. (2006) in the $j=11 \rightarrow 10, j=14 \rightarrow 13$ and $j=15 \rightarrow 14$ rotational transitions of $v=0$. The modeling of SiS molecular emissions requires excitation calculations using radiative as well as collisional rate coefficients with the most abundant interstellar species: $\mathrm{He}, \mathrm{H}$, and $\mathrm{H}_{2}$. However, as far as we know, no collisional rates exist for the SiS molecule.

In the absence of available collisional data for the $\mathrm{SiS}$ molecule, rate coefficients of $\mathrm{SiO}$ are frequently used (Schoïer et al. 2005) to estimate the abundance of SiS in molecular clouds. However, recent studies (Lique et al. 2006; Daniel et al. 2006) have shown that the excitation of the molecule is really dependent on the set of collisional rate coefficients used, and that inaccurate rates may lead to important errors in the determination of the molecular abundance in molecular clouds. It is thus of great importance to provide a more realistic determination of $\mathrm{SiS}$ collisional rate coefficients with interstellar species $\mathrm{He}$ and $\mathrm{H}_{2}$. In this paper, we focus on calculations of rate coefficients for rotational excitation of $\mathrm{SiS}$ by $\mathrm{He}$ for temperatures ranging from 5 to $200 \mathrm{~K}$ using accurate theoretical methods. Hence, collisional cross sections, based on a potential energy surface (PES) determined using highly-correlated coupled clusters methods, were computed using close coupling quantum scattering methods.

We have considered rotational excitation of the 26 first rotational levels of SiS. In addition to providing useful data for observations interpretation, this work is also a first step of the thorough theoretical study of ro-vibrational excitation rate coefficients of $\mathrm{SiS}$ by collision with $\mathrm{He}$ ( $\mathrm{SiS}$ was also found in vibrationally excited states in interstellar gas, Turner 1987) and, in the near future, with $\mathrm{H}_{2}$. We expect that the present results will be relevant for interpreting the data that extremely powerful instruments, such as Herschel but also ALMA, will provide in the next few years.

The paper is organized as follows: Sect. 2 describes the ab initio calculation of the potential energy surface, Sect. 3 provides a rapid description of the theory and calculations and in Sect. 4 we present and discuss our results.

\section{Potential energy surface}

The ground electronic state of the SiS-He van der Waals system is a singlet state with ${ }^{1} \mathrm{~A}$ ' symmetry. The SiS-He "rigid rotor" PES is described by the two Jacobi coordinates : $R$, the distance from the center of mass of $\mathrm{SiS}$ to the He atom and $\theta$, the angle between $\boldsymbol{R}$ and the SiS bond axis, $\boldsymbol{r}(\theta=0$ : He adjacent to the Si atom; $\theta=180$ : He adjacent to the sulfur atom). The $\mathrm{SiS} r$-distance was frozen at its experimental equilibrium distance, $r_{\mathrm{e}}=3.6459$ bohr (Huber \& Herzberg 1979). As shown by Lique et al. (2007), in the particular case of the CS-He system, the 2D-PES calculated at the frozen equilibrium $r$-distance and the full 3D-PES averaged over the vibrational ground state of a heavy diatomic molecule, are very similar, and the influence of using one or the other in the dynamical calculation is very small. 


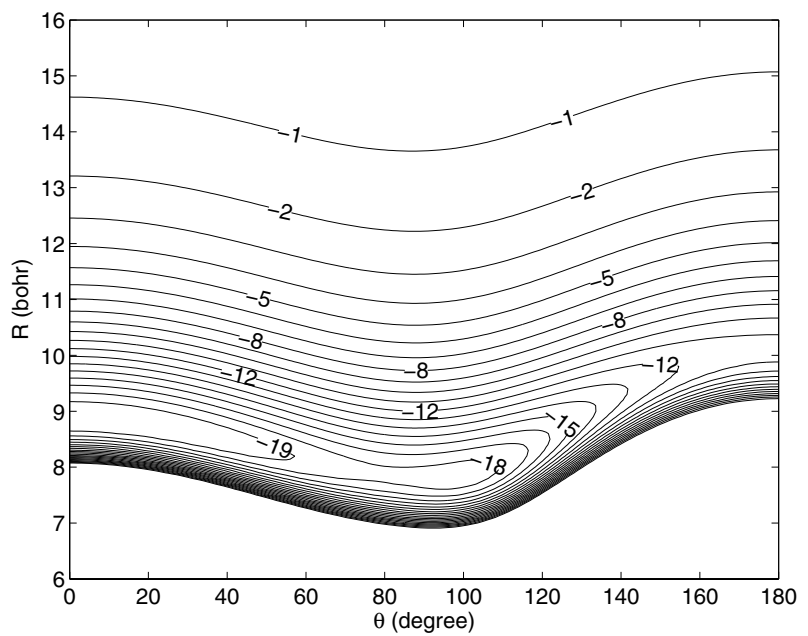

Fig. 1. Contour plot of the SiS-He PES as a function of $R$ and $\theta$ with the $\mathrm{SiS}$ bond distance fixed at $r_{\mathrm{e}}=3.6459 \mathrm{bohr}$. The energies are in $\mathrm{cm}^{-1}$ with a distance of $1 \mathrm{~cm}^{-1}$ between two adjacent contours, and the zero of energy is taken as that of the $\mathrm{SiS}+\mathrm{He}$ asymptote.

Consequently, in the present case we expect that restricting $r$ to its equilibrium distance will introduce very little error in the calculated inelastic cross sections and rate coefficients.

The PES was calculated using the supermolecular approach based on a single- and double-excitation coupled cluster method (CCSD) (Hampel et al. 1992) with perturbative contributions from connected triple excitations computed as defined by Watts et al. (1993) (CCSD(T)). The ab initio surface was calculated using the MOLPRO package (2002). For all three atoms, we used the standard correlation-consistent polarized-valence-quadruple zeta sets of Dunning (1989) (cc-pVQZ) augmented with the diffuse functions of $s, \mathrm{p}, \mathrm{d}, \mathrm{f}$, and $\mathrm{g}$ symmetries by Kendall (1992) (aug-cc-pVQZ). This basis set was further augmented by the $[3 \mathrm{~s} 3 \mathrm{p} 2 \mathrm{~d} 2 \mathrm{f} 1 \mathrm{~g}]$ bond functions optimized by Cybulski \& Toczylowski (1999) and placed at mid-distance between the He atom and the CS center of mass. At all geometries, the basis set superposition error (BSSE) was corrected by using the Boys \& Bernardi (1970) counterpoise procedure:

$V(R, \theta)=E_{\mathrm{SiS}-\mathrm{He}}(R, \theta)-E_{\mathrm{SiS}}(R, \theta)-E_{\mathrm{He}}(R, \theta)$

where the energies of the SiS and He subsystems are computed with the full (three atoms plus bond functions) basis set. For such a van der Waals system, where the ground state is well described by a dominant configuration at all computed geometries, this level of theory is expected to yield reliable results.

Using the computational scheme described above, a total of 615 geometries was computed. The radial scattering coordinate $R$ was assigned values from $4.0 a_{0}$ to $16.0 a_{0}$ by steps of $0.25 a_{0}$, and the angular grid was uniform with a 15 degree spacing from 0 to 180 degrees. At short atom-diatom separation, additional geometries were computed in such a way that the interaction energy becomes at least $10000 \mathrm{~cm}^{-1}$ at any angle. A contour plot of the potential is shown in Fig. 1. For this van der Waals system, the global minimum in the interaction energy was found to be $-19.97 \mathrm{~cm}^{-1}(R=8.87 \mathrm{bohr}, \theta=0$ degree $)$.

An analytic representation of the 2D PES, suitable for dynamics calculations, was obtained using the fitting procedure described by Eqs. (19)-(22) of Werner et al. (1988) for the CN-He system, in the particular case of $r=r_{\mathrm{e}}$. Over the entire grid, the mean difference between the analytic fit and the ab initio calculations is $0.5 \%$.

\section{Collision dynamics}

The fitted SiS-He PES was used to determine rotational excitation and de-excitation cross sections of $\mathrm{SiS}$ molecules by $\mathrm{He}$ atoms.

The full close coupling approach (CC), first introduced by Arthurs \& Dalgarno (1960), and described in Flower (1990), was used to calculate the cross sections involving the 26 first rotational levels. The integral cross sections at each kinetic energy $E_{k}$ are obtained by summing the partial cross sections over $J$, the total angular momentum, until convergence is reached.

All the scattering calculations were performed using the MOLSCAT code (Hutson \& Green 1994). The rotational energy levels of the ${ }^{28} \mathrm{Si}^{32} \mathrm{~S}$ molecule were computed using the experimental spectroscopic constants of Tiemann et al. (1977). The calculations were carried out using the propagator of Manolopoulos (1986). The reduced mass of the system is 3.7528. The analytic PES was used to obtain the usual Legendre expansion:

$V\left(r=r_{\mathrm{e}}, R, \theta\right)=\sum_{\lambda=0}^{\lambda_{\max }} V_{\lambda}(R) P_{\lambda}(\cos \theta)$.

From an ab initio grid containing 13 values of $\theta$, we were able to include terms up to $\lambda_{\max }=12$.

The propagation parameters were tested in order to obtain convergence of the cross sections for total energies up to $1500 \mathrm{~cm}^{-1}$. Typically, the minimum and maximum integration distances are $R_{\min }=2.75 \mathrm{bohr}$ and $R_{\max }=40 \mathrm{bohr}$, respectively. We extended the rotational basis sufficiently to ensure convergence of the inelastic cross sections for $j, j^{\prime} \leq 25$. At $1500 \mathrm{~cm}^{-1}$, where the transitions among the first 26 rotational levels were used in computing rate coefficients, the calculations included 36 rotational levels.

We carefully spanned the energy ranges to take the presence of resonances into account. The energy steps are $0.1 \mathrm{~cm}^{-1}$ below $50 \mathrm{~cm}^{-1}, 0.2 \mathrm{~cm}^{-1}$ from 50 to $100 \mathrm{~cm}^{-1}, 0.5 \mathrm{~cm}^{-1}$ from 100 to $150 \mathrm{~cm}^{-1}$, 1 from 150 to $250 \mathrm{~cm}^{-1}, 2$ from 250 to $350 \mathrm{~cm}^{-1}, 4$ from 300 to $400 \mathrm{~cm}^{-1}$, 10 from 400 to $500 \mathrm{~cm}^{-1}, 50$ from 500 to $1000 \mathrm{~cm}^{-1}$, and 100 from 1000 to $1500 \mathrm{~cm}^{-1}$. Convergence of the integration was carefully tested. Calculations up to $1500 \mathrm{~cm}^{-1}$ allow us to determine rates up to $200 \mathrm{~K}$ for the first 26 levels (up to $j=25$ ).

\section{Results}

\subsection{Cross sections}

Figure 2 illustrates the energy dependence of the collisional deexcitation cross sections obtained from the present CC calculations for a few selected rotational levels. The de-excitation cross sections are almost decreasing functions of the energy. For collision energies below $30 \mathrm{~cm}^{-1}$, many resonances are found. These are a consequence of the quasibound states arising from tunneling through the centrifugal energy barrier (shape resonances), or from the presence of an attractive potential well at the linear geometry that allows the He atom to be temporarily trapped into the well and hence quasibound states to be formed (Feshbach resonances) before the complex dissociates (Smith et al. 1979; Christoffel \& Bowman 1983). Owing to the small energy spacing between the SiS rotational levels compared to the potential well depth, both types of resonance may be found in the same energy range. 


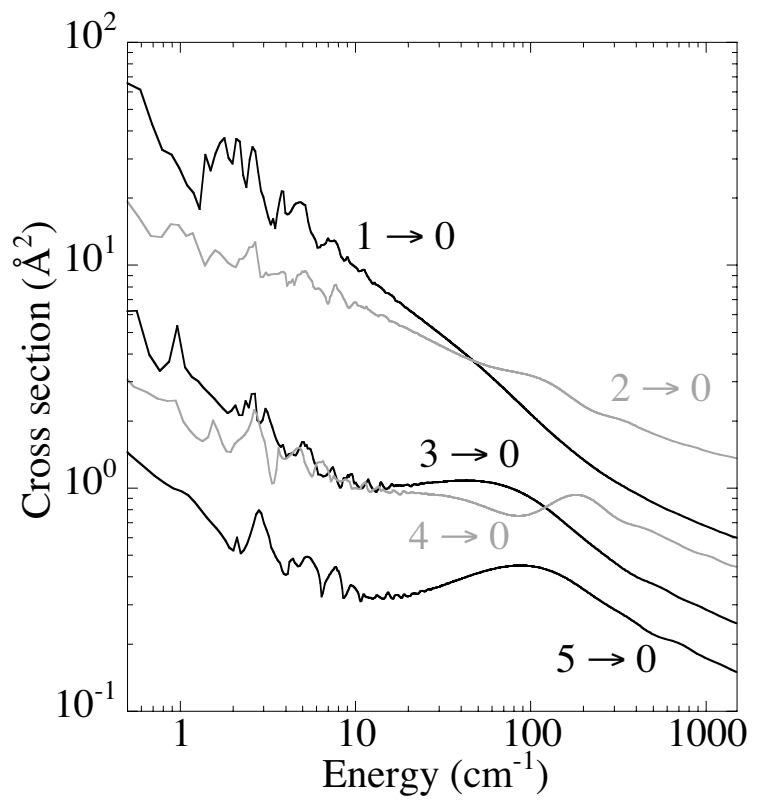

Fig. 2. Rotational de-excitation cross sections of $\mathrm{SiS}$ in collision with $\mathrm{He}$ as a function of the relative kinetic energy. Black lines: odd $\Delta j$ transitions; grey lines: even $\Delta j$ transitions.

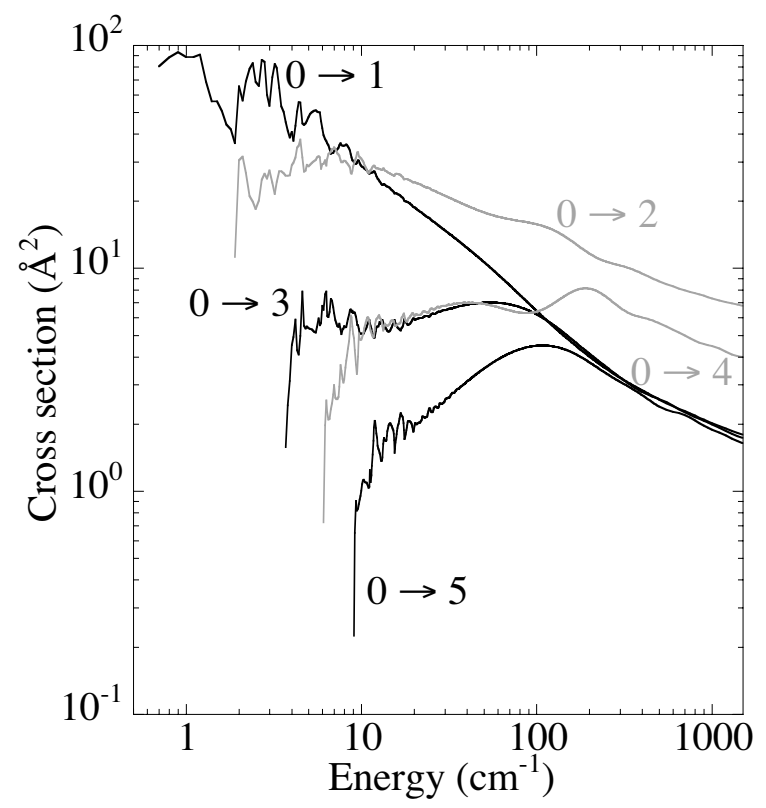

Fig. 3. Rotational excitation cross sections of SiS in collision with $\mathrm{He}$ as a function of the relative kinetic energy. Black lines: odd $\Delta j$ transitions; grey lines: even $\Delta j$ transitions.

The de-excitation cross sections shown in Fig. 3 decrease with increasing $\Delta j$ for a given initial $j$ state and, outside the energy region of resonances, exhibit a propensity in favor of transitions with even $\Delta j$ due to the near-homonuclear symmetry of the potential energy surface. This effect has been seen both experimentally (for NO-Ar collisions, Andresen et al. 1982) and theoretically (for CO-He collisions, Chapman \& Green 1977), and was first explained by Brumer (1974), and later by McCurdy $\&$ Miller (1977). The features shown in the excitation cross sections (see Fig. 4) may be understood from the detailed balance equation that relates excitation and de-excitation cross sections.

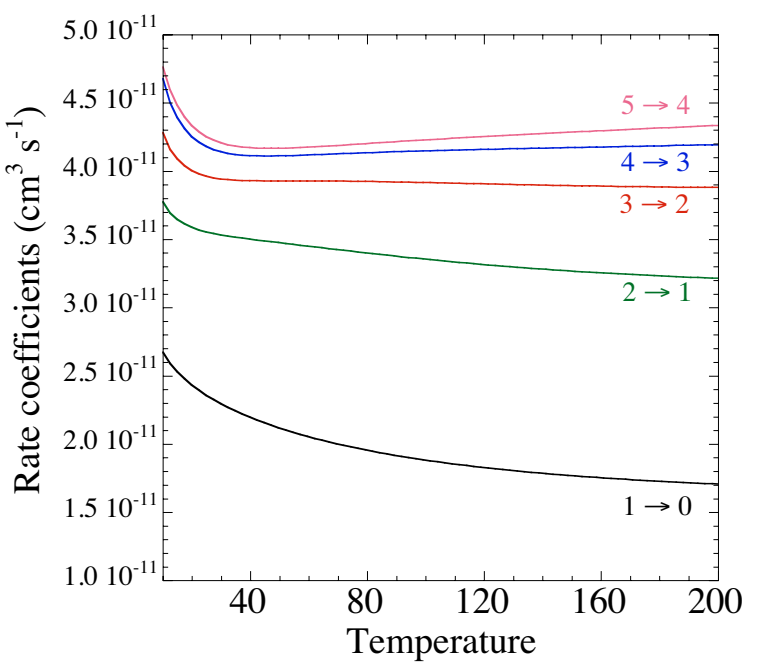

Fig. 4. Temperature variation of the de-excitation rate coefficients for the first $\Delta j=j^{\prime}-j=-1$ transitions.

\subsection{Rate coefficients}

From the rotationally inelastic cross sections $\sigma_{j \rightarrow j^{\prime}}\left(E_{k}\right)$, one can obtain the corresponding thermal rate coefficients at temperature $T$ by an average over the collision kinetic energy $E_{k}$. The rate coefficients are the Boltzmann thermal average at temperature $T$ of collision cross sections $\sigma_{j \rightarrow j^{\prime}}$ :

$k_{j \rightarrow j^{\prime}}(T)=\left(\frac{8 k_{\mathrm{B}} T}{\pi \mu}\right)^{\frac{1}{2}}\left(\frac{1}{k_{\mathrm{B}} T}\right)^{2} \times \int_{0}^{\infty} E_{k} \sigma_{j \rightarrow j^{\prime}}\left(E_{k}\right) \mathrm{e}^{\frac{-E_{k}}{k_{\mathrm{B}} T}} \mathrm{~d} E_{k}$

where $k_{\mathrm{B}}$ is the Boltzmann constant. We have obtained by energy averaging the de-excitation rate coefficients for the 25 first rotational levels from the $\mathrm{CC}$ cross sections. The representative variation with temperature of the rate coefficients from an initial level $j$ to a final level $j^{\prime}$ are shown in Figs. 4 and 5 for $\Delta j=j^{\prime}-j=-1$ and -2 , respectively.

We report in Fig. 6 the de-excitation rate coefficients as a function of $j$ for selected $\Delta j=j^{\prime}-j$ transitions. It is seen that: (i) for a given $\Delta j$ the rate coefficients increase with increasing $j$, the effect being more pronounced for low $j$ values; and (ii) the rate coefficients decrease with increasing $\Delta j$, which is the usual trend. As found for the cross sections, the rate coefficients display the same propensity toward transitions with even $\Delta j$. This is illustrated in Fig. 7, which shows the de-excitation rate coefficients at $T=100 \mathrm{~K}$ out of the $j=10$ level. This propensity may have important astrophysical consequences such as population inversion if the local thermodynamical equilibrium (LTE) conditions are not met.

The complete set of rate coefficients are published in the electronic version of the paper and on our WEB site basecol (wWW.obspm.fr/basecol).

\section{Discussion}

From a quantum scattering calculation we have investigated rotational excitation of SiS in collision with He. The calculations are based on a new accurate 2D PES. From the calculated cross sections, rate coefficients involving the lowest 26 levels of SiS were determined for temperatures ranging from 5 to $200 \mathrm{~K}$. The results show a $\Delta j$ even propensity, in particular for $|\Delta j|=2$ transitions. This propensity can have important astrophysical consequences as a $\Delta j$ even propensity can amplify population inversion if LTE is not realized (Wernli et al. 2006). The $\Delta j$ even 


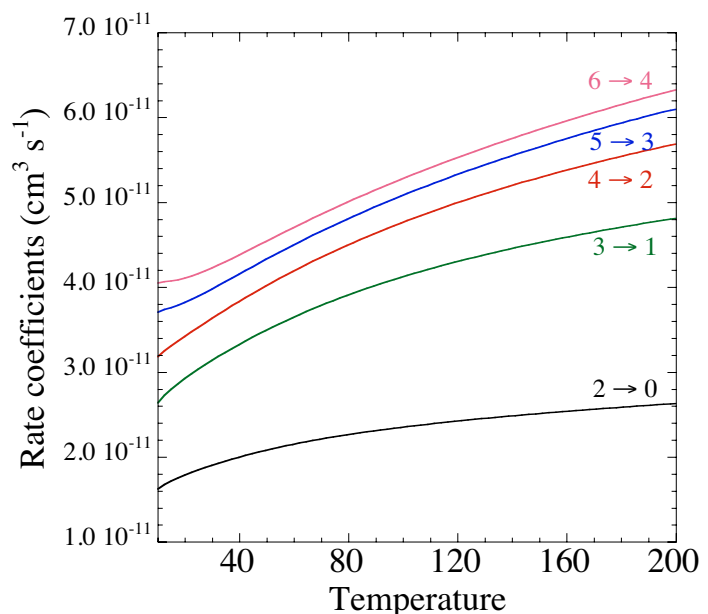

Fig. 5. Temperature variation of the de-excitation rate coefficients for the first $\Delta j=j^{\prime}-j=-2$ transitions.

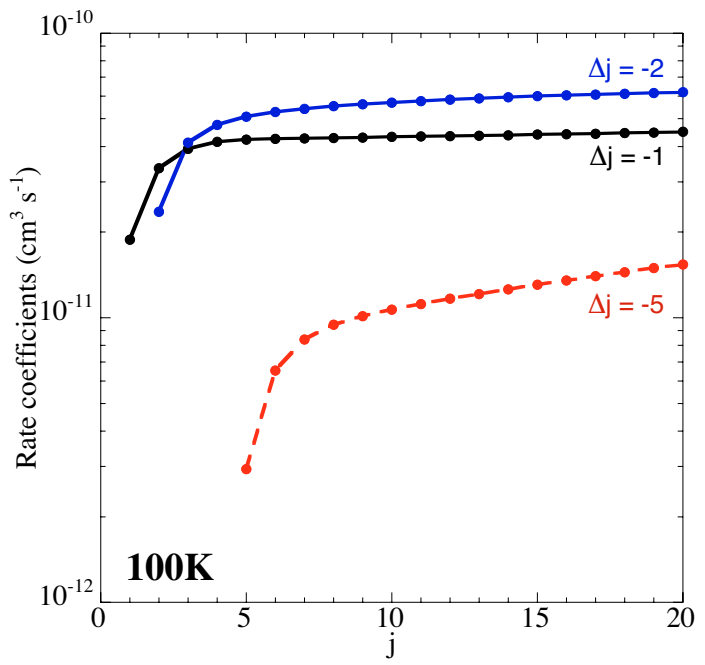

Fig. 6. De-excitation rate coefficients at $100 \mathrm{~K}$ as a function of $j$ for $\Delta j=j^{\prime}-j=-1,-2,-5$.

propensity is opposite to that found for collisional excitation of $\mathrm{SiO}$ by He (Dayou \& Balança 2006). It is therefore important to point out that our results refute the recommendation of using $\mathrm{SiO}$ rate coefficients for $\mathrm{SiS}$ (Schöier et al. 2005).

Finally, as the dominant collision partner in the interstellar medium is $\mathrm{H}_{2}$, it is often proposed to use He-rate coefficients with the appropriate reduced mass correction as a first estimate of rate coefficients with $\mathrm{H}_{2}$ (Schöier et al. 2005). Some caution may be exercised concerning this approximation as recent results on rotational excitation of CO (Wernli et al. 2006) and SO (Lique et al. 2007) reveal that rate coefficients for collisions with para$\mathrm{H}_{2}(j=0)$ can be up to 3 times larger or smaller than those for collisions with $\mathrm{He}$, depending on the selected transition. It is therefore crucial to extend the calculations, both of the PES and of the cross sections, to the $\mathrm{SiS}-\mathrm{H}_{2}$ system. These calculations are presently in progress (Lique 2007).

Acknowledgements. We would like to acknowledge N. Feautrier for her continuing interest in this work and for the fruitful discussions. We also acknowledge G. Dhont for making his fitting code of the potential energy surface available to us. The calculations of ab initio potential energy surfaces were performed on the parallel machine MPOPM of Paris Observatory, and all the scattering calculations were performed at the IDRIS-CNRS center (Institut de Développement et des Ressources en Informatique Scientifique du Centre National de la Recherche Scientifique) under projects 060883 and 070883 . This study is supported in part

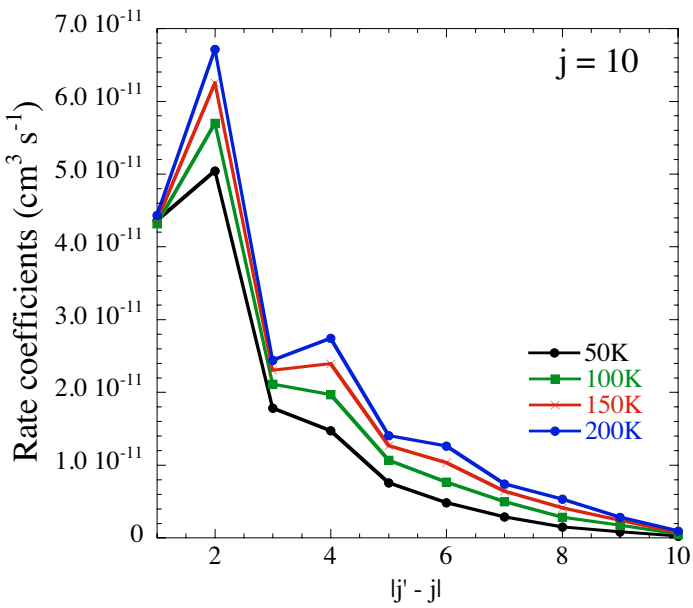

Fig. 7. De-excitation rate coefficients at $100 \mathrm{~K}$ from the $j=10$ level as a function of $|\Delta j|$

by the European Community as human potential Programme under contract MCRTN 512302, Molecular Universe. F.L. would like to acknowledge financial support from the U. S. National Science Foundation under grant CHE-0413743. The authors thank the referee for helpful comments.

\section{References}

Andresen, P., Joswig, H., Pauly, H., \& Schinke, R. 1982, J. Chem. Phys., 77, 2204

Arthurs, A. M., \& Dalgarno, A. 1960, Proc. Roy. Soc. A, 256, 540

Bieging, J. H., \& Nguyen-Quang-Rieu 1989, ApJ, 343, L25

Boys, S. F., \& Bernardi, F. 1970, Mol. Phys., 19, 553

Brumer, P. 1974, Chem. Phys. Lett., 28, 345

Chapman, S., \& Green, S. 1977, J. Chem. Phys., 67, 2317

Christoffel, K. M., \& Bowman, J. M. 1983, J. Chem. Phys., 78, 3952

Cybulski, S. M., \& Toczylowski, R. R. 1999, J. Chem. Phys., 111, 10520

Daniel, F., Cernicharo, J., \& Dubernet, M.-L. 2006, ApJ, 648, 461

Dayou, F., \& Balança, C. 2006, A\&A, 459, 297

Dunning, T. H. 1989, J. Chem. Phys., 90, 1007

Flower, D. 1990, Molecular collisions in the interstellar medium, Cambridge Astrophysics Series

Fonfría Expósito, J. P., Agúndez, M., Tercero, B., Pardo, J. R., \& Cernicharo, J. 2006, ApJ, 646, L127

Henkel, C., Matthews, H. E., \& Morris, M. 1983, ApJ, 267, 184

Huber, K. P., \& Herzberg, G. 1979, Constants for diatomic molecules (Van Nostrand Reinhold Company)

Kendall, R. A., Dunning, T. H., \& Harrison, R. J. 1992, J. Chem. Phys., 96, 6796 Hutson, J. M., \& Green, S. 1994, MOLSCAT computer code, version 14 (Collaborative Computational Project No. 6 of the Science and Engineering Research Council, UK)

Hampel, C., Peterson, K., \& Werner H.-J 1992, Chem. Phys. Lett., 190, 1

Lique, 2007, personal communication

Lique, F., Cernicharo, J., \& Cox, P. 2006, ApJ, 653, 1342

Lique, F., \& Spielfiedel, A. 2007, A\&A, 462, 1179

McCurdy, C. W., \& Miller, W. H. 1977, J. Chem. Phys., 67, 463

Morris, M., Gilmore, W., Palmer, P., Turner, B. E., \& Zuckerman, B. 1975, ApJ, 199, L47

Manolopoulos, D. E. 1986, J. Chem. Phys., 85, 6425

MOLPRO is a package of ab initio programs written by

Werner, H.-J., \& Knowles, P. J. with contributions from Almlöf, J., Amos, R. D., Deegan, M. J., Elbert, S. T., Hampel, C., Meyer, W., Peterson, K., Pitzer, R., Stone, A. J., Taylor, P. R., Lindh, R., Mura, M. E., \& Thorsteisson T.

Olofsson, H., Johansson, L. E. B., Hjalmarson, A., \& Nguyen-Quang-Rieu 1982, A\&A, 107, 128

Schöier, F. L., van der Tak, F. F. S., van Dishoeck, E. F., \& Black, J. H. 2005, A\&A, 432, 369

Smith, L. N., Malik, D. J., \& Secrest, D. 1979, J. Chem. Phys., 71, 4502

Tiemann, E., Renwanz, E., Hoeft, J., \& Törring, T. 1977, Z. Naturforsh., 27a, 1566

Turner, B. E. 1987, A\&A, 183, L23

Watts, J. D., Gauss, J., \& Bartlett, R. J. 1993, J. Chem. Phys., 98, 8718

Werner, H.-J., Follmeg, B., \& Alexander, M. H. 1988, J. Chem. Phys., 89, 3139

Wernli, M., Valiron, P., Faure, A., et al. 2006, A\&A, 446, 367 